\title{
Analysis of Switching Dc-dc Converters Using a Grid-point Approach
}

\author{
F. H. F. Leung*, P. K. S. Tam ${ }^{* *}$, D. P. Kwok ${ }^{* * *}$, C. Y. Cheung ${ }^{* * *}$ \\ - Department of Electronic Engineering, Hong Kong Polytechnic, Hung Hom - Hong Kong \\ Phone: 852-766-6224; fax: 852-362-8439; e-mail: enfrank@hkpcc.hkp.hk \\ ** Department of Electronic Engineering, Hong Kong Polytechnic, Hung Hom - Hong Kong \\ Phone: 852-766-6238; fax: 852-362-8439; e-mail: enptam@hkpcc.hkp.hk \\ -.* Department of Electronic Engineering, Hong Kong Polytechnic, Hung Hom - Hong Kong \\ Phone: 852-766-6208; fax: 852-362-8439; e-mail: enkwokdp@hkpcc.hkp.hk \\ *... Department of Electronic Engineering, Hong Kong Polytechnic, Hung Hom - Hong Kong \\ Phone: 852-766-6251; fax: 852-362-8439; e-mail: may@encserver.en.hkp.hk
}

\begin{abstract}
A switching dc-dc Converter in closed-loop steady-state constitutes an operating point in the space of parameters describing its model. The inadequacy of conventional design of regulated converters, which is based on the assumption of smallsignal perturbations about a single operating point, is addressed. A systematic and general modelling based on a grid-point approach is thus proposed. Under this modelling approach, multiple operating points within the parameter space are reduced to some defined grid-points describing the complete operation space of a regulated converter subjected to large-signal disturbances. The operation subspace of a given grid-point is analyzed. An example based on a PWM-type boost converter is to be given.
\end{abstract}

\section{INTRODUCTION}

Switching dc-dc converters, being the power processing units of switch mode power supplies, have earned much research interest in the past decades due to their high efficiency and power density [1][2]. Practically, a converter is only an open-loop plant for which a controller (or simply a compensator) is designed to close the loop in order to achieve the necessary regulation of output power against any disturbances to the system. The design of controller is usually based on a model of the converter previously defined. Much work on analysis and modelling of switching dc-dc converters was done in the past [3][4].

Converters are highly non-linear by nature. Besides, the parameters of the converter model are uncertain and subjected to inevitable and significant perturbations during operation [5]. The usual modelling approaches using averaging and small-signal linearization techniques are often found ineffective or inadequate in many applications when the range of operation and/or the signal of disturbances are large. This paper aims at exploring and tackling the issue of modelling regulated switching dc-dc converters and proposing a systematic and general approach in large-signal.

The output voltage $v_{o}$ of a converter is regulated by some control signal (duty ratio $d$ or modulated frequency $f$ of the switch). In its steady-state, a converter has an operating point in the parameter space. A small-signal linear model based on this operating point can

This work was supported by the Hong Kong Polytechnic Research Grant for the project of grant number $340 / 443$. be derived by averaging and linearization [1]. However, when the converter is operating, the parameters of the converter model are affected by the disturbances on input line voltage $v_{i}$, output current $i_{o}$ (output load), and other minor factors such as EMI and stray effects. In other words, converter spans multiple operating points in the parameter space during operation, termed the operation space of the converter. Conventional controller design assumes that the disturbances are small such that the single operating point model obtained through averaging and linearization is still an acceptable representation of the converter. From another point of view, on closing the loop, the maximum deviation of the parameters from that single operating point allowable in accordance with some design criteria (e.g. stability of the closed-loop system) has covered the operation space of the converter based on the specification. A good closed-loop controller is one which allows large values of maximum deviations.

The modelling approach based on single operating point has two major disadvantages: :

1) The assumption can be invalid in many applications, i.e. the maximum parameter deviations that can be tolerated by the controller cannot cover the whole operation space specified.

2) Even though the whole operation space can be covered, the controller may not be robust enough to give satisfactory dynamic responses under large signal disturbances.

To alleviate these disadvantages, a more complete modelling based on a grid-point approach is proposed. For a given operating point (grid-point) in the multiple operating point parameter space, the space spanned by the varying parameters based on some defined criteria is to be analyzed. The information obtained can then be used to determine other grid-points until the parameter space spanned by all grid-points has cover the operation space of the converter. In this way, the large operation space of the converter is reduced to some defined grid-points, and any large-signal disturbances causing large parameter variations can be localized to some small-signal disturbances around one of the grid-points.

The single operating point small-signal model based on averaging and linearization together with its limitation will be discussed in Section II. The grid-point modelling approach will be detailed in Section III. In Section IV, an application example based on a PWMtype boost converter will be given. 


\section{SMALL-SIGNAL LINEAR MODEL}

When the perturbations to a regulated switching dc-dc converter (in closed-loop) are small, the controller can be designed based on a small-signal linear model of the converter. A controller of transfer function $H(s)$ is to be designed to close the loop so as to achieve the necessary control. The converter model is represented by a linear control-to-output transfer function $G(s)$. For the PWM-type converter, $G(s)$ is given by the ratio of the perturbed output voltage $\bar{v}_{0}(s)$ to the perturbed duty-ratio $\tilde{d}(s)$ of the switch. A block diagram representing this small-signal closed-loop system is shown in Fig. 1. Many methods are available to derive the transfer function $\tilde{v}_{0}(s) / \tilde{d}(s)$. Among them, the most popular one is the state-space averaging approach [3][6] which can be summarized into the following steps:

1) State-variable description for each circuit state.

2) Averaging the state-variable description using the duty ratio $d$.

3) Introducing small ac perturbations and separation in ac and dc components.

4) Transformation of the ac state-equations into $s$-domain to solve for the control-to-output transfer function.

In step 2, the averaged state-space model is derived and represented by the state matrix and vectors $(A, B, C)$. Small ac perturbations (represented by $\because$ ) are introduced onto the dc steady-state quantities (represented by uppercase letters) in step 3 . Therefore, the statevariable $x$, output voltage $v_{o}$ and duty-ratio $d$ are given by:

$$
\begin{aligned}
& x=X+\bar{x} \\
& v_{o}=V_{o}+\tilde{v}_{o} \\
& d=D+\tilde{d}
\end{aligned}
$$

so that the state-space model can be represented as follows:

$$
\begin{aligned}
& \dot{\tilde{x}}= A \tilde{x}+\left[\left(A_{1}-A_{2}\right) X+\left(B_{1}-B_{2}\right) V_{1}\right] \tilde{d} \\
&+ \text { terms containing products of } \tilde{x} \text { and } \tilde{d} \\
& \tilde{v}_{o}=C \tilde{x}+\left[\left(C_{1}-C_{2}\right) X\right] \tilde{d}
\end{aligned}
$$

where the subscripts 1 and 2 denotes the switch state of "on" and "off" respectively. The small-signal control-to-output transfer function is obtained through step 4 by neglecting the terms containing products of $\tilde{x}$ and $\tilde{d}$ in equ. (4). Here, two points should be noted:

1) Converters are non-linear systems. The small-signal model is only an approximate representation obtaining through averaging and linearization by assuming that the perturbed statevariable $\tilde{x}$ and the control signal $\tilde{d}$ are small.

2) During operation, disturbances to the converter (e.g. load and line variations) are inevitable which effectively change the parameters of its model. The control-to-output transfer function is obtained based on an operating point of reflected by certain parameters in the state-matrices and vectors in equ. (4) and (5), and this small-signal model becomes inaccurate when disturbances cause parameter variations.

The problems caused by point 1 depend on those caused by point 2 because the perturbations to $\tilde{\boldsymbol{x}}$ and $\tilde{d}$ follow the shift of operating point dynamically. When the disturbances are constant and sustained, the operating point will shift to a new location and remain steady, with $\bar{x}$ and $\tilde{d}$ to be equal to zero, until new disturbances are added. So the problems of point 1 are on the transient responses but not on the steady-state ones, and the degree of troubles depends on the degree of shifting of operating point in the parameter space.

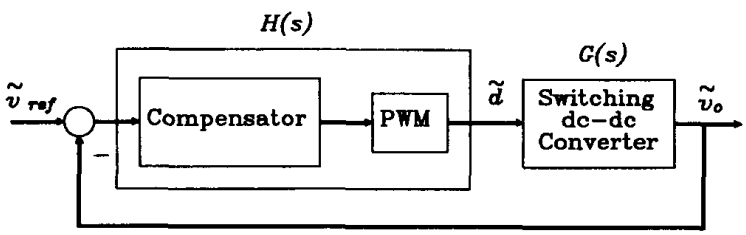

Fig. 1 A block diagram representing the small-signal closed-loop system of regulated converter.

The problems caused by point 2 effectively restrict the degree of parameter variations, or in other words, the extent of shifting of operating point in the parameter space. It is because a new model is associated with a new operating point after shifting, and if the original model before shifting is used to model this new operating point, it is bounded to be inaccurate. The degree of troubles depends on the results caused by this inaccuracy and is tightened when the extent of shifting of operating point increases.

Conventionally, a controller is designed based on a chosen operating point to close the feedback loop such that a larger variation of parameters from the operating point can be allowed (without causing too much troubles due to the inaccuracy of the model on shifting the operating point) when compared with the open-loop converter. The controller is acceptable when the allowable parameter variations have covered the specified range of operation, and is a good one when the controller is so robust that satisfactory responses can be obtained within the specified operation range in the parameter space. Much work on the controller design based on this conventional approach has been reported [7][8]. Nevertheless, when the specification of the regulated converter is harsh such that a wide range of operation is required, e.g. a large fluctuation in the output load and variation in the input line voltage, the conventional approach of modelling and control may not be applicable due to the problems caused by modelling inaccuracy. A more complete modelling approach which tackles this inadequacy is thus needed.

\section{GRID-POINT MODELLING APPROACH}

The modelling approach based on averaging and linearization around a single operating point can be used as a basis for the development of a more complete model for switching dc-dc converters. Based on one operating point, with a designed controller in closed-loop, the allowable parameter space enclosing the operating point such that the disturbances causing parameter variations are regarded small can be investigated. This allowable parameter space depends on the type of the converter and the form of control law, and involves multi-dimensional hyperspaces when the number of varying parameters becomes high. Once an idea about the allowable parameter space spanned by a chosen operating point, or the parameter subspace spanned by a grid-point, is obtained, other gridpoints can be defined then and the parameter subspaces associated with these grid-points can also be investigated until the union of these subspaces covers the operation space of the converter specified. The idea of this grid-point modelling approach is illustrated by a diagram as shown in Fig. 2. In this diagram, $a_{1}$ and $a_{2}$ are variable parameters due to disturbances added to the system, G1, G2, G3 and G4 are grid-points defined on the $\left(a_{1}, a_{2}\right)$ parameter space. The ellipse surrounding each grid-point denotes the boundary inside which the individual parameter subspace is defined based on some criteria (to be further discussed). The rectangle denotes the boundary inside which the operation space of the converter according to its 


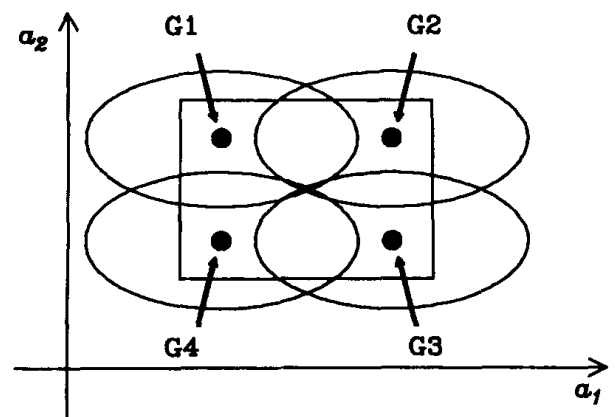

Fig. 2 A diagram illustrating the idea of the grid-point modelling approach.

specification is defined.

In order to derive the parameter subspace spanned by a given gridpoint, the following steps are proposed:

1) For a defined grid-point, with the controller designed according to the small-signal model based on that grid-point, the space of variable parameters derived based on a given criterion is developed.

2) Since the space of variable parameters derived in step 1 has neglected the modelling inaccuracy due to the change of operating point (point 1 and 2 of Section II), the allowable parameter subspace spanned by a grid-point should be a weighted reduction of the space derived in step 1 to ensure that the disturbances causing parameter variation can still be regarded as small signals.

In step 1, the defined criterion can be any arbitrary one based on certain performance measures. If "stability of the closed-loop system based on the given grid-point model" is chosen as the criterion, the Routh criterion or Jury test [9] can be applied to determine the parameter space inside which the closed-loop system is stable under a given controller. If the controller is a digital one, a more general criterion based on the locations of the closed-loop poles can be developed. This is modified from the work of Kaesbauer and Ackermann on the stability boundary of a closed-loop system [10]. The details are discussed in the following two subsections.

\section{A. Boundary of Parameter Space for a Grid-point - the Problem}

Given an operating point of converter model parameter vector $a_{b}$ in the parameter space whose elements are the coefficients of a given linear discrete-time transfer function $G_{o}(z)$ of the plant, a controller associated with this operating point is designed which is represented by a linear discrete-time transfer function $H(z)$. Let $a_{o}$ be subjected to perturbations denoted by the perturbation vector $q$ such that the plant model parameter vector is changed to $a$ given by:

$$
a=a_{o}+q
$$

Let the linear discrete-time transfer function of the converter corresponding to $a$ be given by $G(z, a)$. The closed-loop system formed by the controller and the plant has a characteristic equation given by:

$$
\operatorname{den}\left[G_{c k}(z, a)\right]=1+G(z, a) H(z)=0
$$

where $\operatorname{den}\left[G_{c}(z, a)\right]$ denotes the denominator of the closed-loop transfer function $G_{c d}(z, a)$. The poles of $G_{c l}(z, a)$ can be restricted inside the circle centred at the origin and of radius $\mathbf{k}$ on the $z$-plane $(k \leq 1)$. Since equ. (7) depends on $G(z, a)$ which in turn depends on $a$ (or $q$ ), conversely, any set of poles inside the circle of radius $k$ in the $z$-plane can be mapped to a set of points in the parameter space such that a region of allowed values of $a$ (or $q$ ) can be defined. Thus, the points on the circle of radius $\mathbf{k}$ will define a boundary in the parameter space associated with the grid-point $a_{a}$. So, the problem is: how to determine such a boundary in the parameter space?

An analysis for a given grid-point should be done to investigate the region in the parameter space such that any plant model vector inside the region formed a closed-loop system of poles lying inside the circle of radius $\mathbf{k}$ in the $z$-plane with the control law associated with that grid-point.

\section{B. Boundary of Parameter Space for a Grid-point - the Solution}

Consider equ. (7). The boundary in the parameter space, such that any vector inside the region bounded by it is associated with a closed-loop system of poles lying in the $z$-plane circle of radius $\mathrm{k}$, can be examined by deriving equations in $a$ when the roots of $\operatorname{den}\left[G_{c}(z, a)\right]$ lie on the circle [11]. As far as the set of points on the circle is concerned, the following three cases can be distinguished:

Case 1): $z=\mathbf{k}$;

Using this value of $z$ as a root of $\operatorname{den}\left[G_{c}(z, a)\right]$, we have

$$
\begin{aligned}
& \operatorname{den}\left[G_{c}(k, a)\right]=0 \\
& \Rightarrow c_{1}+\mathbf{g}_{1}^{\mathrm{T}} a=0
\end{aligned}
$$

for some scalar constant $c_{1}$ and constant vector $\mathbf{g}_{\mathbf{1}}$.

Case 2): $z=-\mathrm{k}$;

Similar to Case 1 , using this value of $z$ as a root of $\operatorname{den}\left[G_{c}(z, a)\right]$, we have

$$
\begin{aligned}
& \operatorname{den}\left[G_{c r}(-\mathbf{k}, a)\right]=0 \\
& \Rightarrow c_{2}+g_{2}{ }^{\mathrm{T}} a=0
\end{aligned}
$$

for some scalar constant $c_{2}$ and constant vector $\mathbf{g}_{2}$.

Case 3): $z=k \cdot \exp (j \theta) ; \quad \theta \in(-\pi, \pi)$ and $\theta \neq 0$

Using these values of $z$ as roots of $\operatorname{den}\left[G_{c}(z, a)\right]$, we have

$$
\begin{aligned}
& \operatorname{den}\left[G_{c}(k \cdot \exp (j \theta), a)\right]=0 \\
\Rightarrow & \operatorname{den}\left[G_{c}(k \cdot(\cos (\theta)+\mathrm{jsin}(\theta)), a)\right]=0 \\
\Rightarrow & \left(c_{3}(\theta)+g_{3}(\theta, a)\right)+\mathrm{j}\left(\mathrm{c}_{4}(\theta)+\mathrm{g}_{4}(\theta, a)\right)=0 \\
\Rightarrow & \begin{array}{c}
\mathrm{c}_{3}(\theta)+\mathrm{g}_{3}(\theta, a)=0 \\
\mathrm{c}_{4}(\theta)+\mathrm{g}_{4}(\theta, a)=0
\end{array}
\end{aligned}
$$

for some scalar functions $c_{3}, c_{4}, g_{3}$ and $g_{4}$. Equ. (10) and (11) can be further resolved by eliminating $\theta$ to obatin a linear or non-linear function $\mathrm{g}(\cdot)$ of $a$ such that:

$$
\mathbf{g}(\boldsymbol{a})=0
$$

From the above three cases, it can be seen that every point lying on the boundary the $z$-plane circle of radius $k$ maps to a hypersurface in the parameter space. (This hypersurface is a line or a curve in a 2dimensional parameter space, and is a plane or a surface in a 3- 
dimensional parameter space, and so on.) The hypersurface will divide the whole parameter space into two hyperspaces. Based on the above three cases, the idea can be expressed mathematically as:

$$
\begin{aligned}
& \text { Case 1): } z=\mathrm{k} ; \\
& \quad(z=\mathrm{k})-\mathrm{l}_{1} \\
& \Rightarrow \mathrm{Z}_{1}^{+}=\{z \mid z>\mathrm{k}\}-\mathrm{S}_{1}^{+} \text {and } \mathrm{Z}_{1}^{-}=\{z \mid z<\mathrm{k}\}-\mathrm{S}_{1}^{-} \\
& \text {Case 2): } z=-\mathrm{k} ; \\
& \quad(z=-\mathrm{k})-\mathrm{l}_{2} \\
& \Rightarrow \mathrm{Z}_{2}^{+}=\left\{\left.z\right|^{2} z<-\mathrm{k}\right\}-\mathrm{S}_{2}^{+} \text {and } \mathrm{Z}_{2}^{-}=\{z \mid z>-\mathrm{k}\}-\mathrm{S}_{2}^{-} \\
& \text {Case 3): } z=\mathrm{k} \cdot \exp (\mathrm{j} \theta) ; \quad \theta \in(-\pi, \pi) \text { and } \theta \neq 0 \\
& \quad(z=\mathrm{k} \cdot \exp (\mathrm{j} \theta))-\mathrm{l}_{3} \\
& \Rightarrow \mathrm{Z}_{3}^{+}=\{z|| z \mid>\mathrm{k}\}-\mathrm{S}_{3}^{+} \text {and } \\
& \quad \mathrm{Z}_{3}^{-}=\{z|| z \mid<\mathrm{k}\}-\mathrm{S}_{3}^{-}
\end{aligned}
$$

where the symbol "م" denotes a mapping from either direction, $1_{1}, 1_{2}$, $l_{3}$ are hypersurfaces in the parameter space containing $\alpha$, and $S_{1}^{+}, S_{1}^{-}$, $\mathrm{S}_{2}{ }^{+}, \mathrm{S}_{2}{ }^{-}, \mathrm{S}_{3}{ }^{+}, \mathrm{S}_{3}{ }^{-}$are hyperspaces divided by the associated hypersurfaces. From the expressions of she above three cases, it can be seen that the intersection given by $\left(Z_{1}^{-} \cap Z_{2} \cap Z_{3}\right)$ is the region of circle of radius $\mathbf{k}$ in the $z$-plane, and the corresponding parameter space containing $a$ is given by:

$$
S^{-}=\left(S_{1}{ }^{\circ} \cap S_{2}{ }^{\circ} \cap S_{3}\right)
$$

$S^{-}$is thus the parameter space spanned by the grid-point $a_{e}$. In particular, when $k=1$, the parameter space of $S^{-}$is that obtained by using Routh criterion or Jury test.

\section{Forming the Grid-point Model}

The parameter space derived based on a given criterion from a small-signal model associated with a grid-point of the converter, once known, can be reduced by a weighting factor to obtain the operation subspace of the grid-point in the parameter space. Since the operation subspace of a grid-point can be found and the operation space of the converter is well-defined (based on the design specification), we can define other grid-points (i.e. operating points in other sustained perturbed conditions) in the parameter space such that the union of all operation subspaces associated with all grid-points encloses the operation space. This is somewhat akin to the partitioning approach of non-linear system control [12][13]. Under this approach, the operation space is reduced to a set of grid-points and large-signal disturbances changing the operating points are localized to small-signal disturbances around a grid-point. It can be seen that in order to complete the control, a strategy to determine the gridpoint best representing the converter during operation has to be derived. Since the modelling, but not the control, of switching dc-dc converters is the main concern of this paper, the strategy of determining the best grid-point is not to be further discussed.

\section{APPLICATION EXAMPLE}

A PWM-type boost converter can be used as an example to illustrate the grid-point modelling approach. The schematic of the boost converter is shown in Fig. 3. Suppose the unregulated converter is subjected to a line variation from $7 \mathrm{~V}$ to $16 \mathrm{~V}$ and load variation from $4 \Omega$ to $32 \Omega$. A simple digital proportional controller of gain $K_{p}$ and sampling frequency $\mathrm{T}$ of $1 \mathrm{kHz}$ is to be designed to

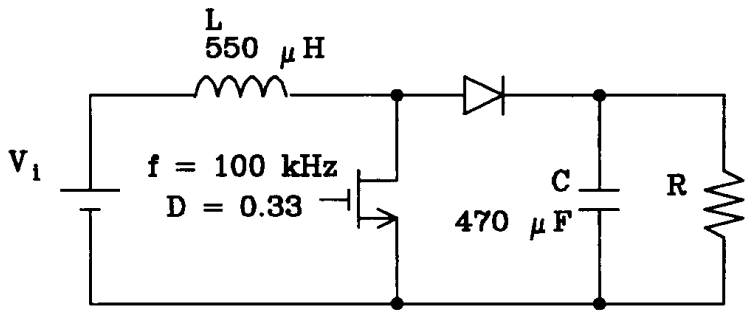

Fig. 3 Schematic of the boost converter.

close the feedback loop for regulation. For simplicity, only the input line voltage $V_{i}$ and output load $R$ are regarded as variable parameters affected by disturbances to the system. The other parameters are assumed constant. To develop the grid-point model, the control-tooutput transfer function in $s$-domain should firstly be known. Then, by using the bilinear transform:

$$
s=\frac{2}{T} \cdot \frac{z-1}{z+1}
$$

the discrete-time control-to-output transfer function $G(z)=\tilde{v}_{0}(z) / \tilde{d}(z)$ is derived. It can be shown that $G(z)$ is given by the following expression:

$$
\begin{aligned}
G(z)= & \frac{V T T}{R L C(1-D)^{2}} \cdot \frac{\left[\left(R T(1-D)^{2}-2 L\right) z^{2}+2 R T(1-D)^{2} z+\right.}{\left(4+\frac{2 T}{C R}+\frac{(1-D)^{2} T^{2}}{C L}\right) z^{2}+} \\
& \frac{R T(1-D)^{2}+2 L}{\left(\frac{2(1-D)^{2} T^{2}}{C L}-8\right)+4-\frac{2 T}{C R}+\frac{(1-D)^{2} T^{2}}{C L}}
\end{aligned}
$$

The stability of the closed-loop system when $V_{t}$ and $R$ are varying about a given grid-point is chosen as the criterion to determine the parameter space spanned by a grid-point. The method proposed in Section III with $\mathrm{k}=1 \mathrm{can}$ be applied. By obtaining $\operatorname{den}\left[G_{c}\left(z, V_{i}, R\right)\right]$ based on a proportional controller of gain $K_{p}$, it can be shown that the boundary of parameter space derived from a small-signal model associated with a grid-point can be acquired from the following three cases:

Case 1): $z=1$;

Using this value of $z$ as a root of $\operatorname{den}\left[G_{c k}\left(z, V_{i}, R\right)\right]$, we have

$$
\begin{aligned}
& R=0 \\
& V_{i}=-(1-\mathrm{D})^{2} / K_{p}
\end{aligned}
$$

Case 2): $z=-1$;

Using this value of $z$ as a root of $\operatorname{den}\left[G_{c}\left(z, V_{b}, R\right)\right]$, we have

$$
R=0
$$

Case 3): $z=\exp (\mathrm{j} \theta) ; \quad \theta \in(-\pi, \pi)$ and $\theta \neq 0$

Using these values of $z$ as roots of $\operatorname{den}\left[G_{c c}\left(z, V_{b} R\right)\right]$, we have

$$
V_{i}=(1-\mathrm{D})^{2} / K_{p}
$$

The parameter space to be derived is the region enclosed by the lines given by equ. (16) to (19). With reference to the operation space of the converter, a first grid-point of $R=5 \Omega$ and $V_{i}=8 \mathrm{~V}$ is chosen 
under a designed $K_{p}$ of 0.02 . To ensure that the disturbances to the system be small-signals around the grid-point in order to alleviate the non-linear effect, a reduction weighting factor is introduced to obtain the operation subspace associated with this grid-point. In this particular example, since one boundary of $R$ is at infinity, the weighting factor is introduced to $V_{i}$ only. For $\mathrm{D}=0.33$, the boundary of $V_{i}$ given by equ. (17) and (19) is $\pm(1-0.33)^{2} / 0.02= \pm 22.4 \mathrm{~V}$. With reference to the grid-point of $8 \mathrm{~V}$, a weighting factor of 0.3 is chosen so that the boundary of the operation subspace is given by:

$$
8 \mathrm{~V} \pm 0.3 \times(22.4 \mathrm{~V}-8 \mathrm{~V})=8 \mathrm{~V} \pm 4.32 \mathrm{~V}
$$

The idea can be represented by a diagram as shown in Fig. 4. With reference to the operation space of the converter and equ. (20), another grid-point of $R=30 \Omega$ and $V_{t}=15 \mathrm{~V}$ is then chosen and a controller of gain $K_{p}=0.015$ is designed. The boundary of $V_{i}$ before reduction is therefore $\pm(1-0.33)^{2} / 0.015= \pm 29.9 \mathrm{~V}$. For a grid-point of $15 \mathrm{~V}$ and a weighting factor of 0.3 , the boundary of the operation subspace corresponding to this grid-point is given by:

$$
15 \mathrm{~V} \pm 0.3 \times(29.9 \mathrm{~V}-15 \mathrm{~V})=15 \mathrm{~V} \pm 4.47 \mathrm{~V}
$$

The union of the operation subspaces of these two grid-points has covered operation space of the converter.

\section{CONCLUSION}

Switching dc-dc converters are highly non-linear system and the parameters of their models are subjected to disturbances during operation. The conventional approach of small-signal modelling based on averaging and linearization about a single-operating point has been discussed together with its shortcomings behind. In summary, the operating point of the converter is shifting in the parameter space and the conventional modelling approach is valid only when the disturbances to the system are small such that the shifting of operating point is not very significant. In view of this inadequacy, a more complete modelling approach using a grid-point concept has been put forward for regulated switching dc-dc converters. By depicting the operation space of the converter into a number of defined grid-points, the large-signal disturbances causing significant changes of operating point can be localized to some smallsignal disturbances causing minor parameter variations around a given grid-point. The steps involved on implementing this modelling approach and the criterion based on which the operation subspace associated with a grid-point is derived have been discussed. It should be noted that as far as the control aspect is concerned, a control strategy is needed to determine the best grid-point representing the converter during operation so as the achieve the optimal regulation. The control aspect under this grid-point modelling approach paves the way for further development.

\section{REFERENCES}

[1] S. Cuk and R. D. Middlebrook, Advances in Switched-mode Power Conversion (Vol. I). Pasadena, Califomia: TESLAco, 1983.

[2] S. Cuk and R. D. Middlebrook, Advances in Switched-mode Power Conversion (Vol. II). Pasadena, California: TESLAco, 1983.

[3] N. Mohan, et. al., Power Electronics: Converters, Applications and Design. New York: John Wiley \& Sons, 1989.

[4] F. C. Lee, High-frequency Resonant, Quasi-resonant, and Multiresonant Converters. Virginia: Virginia Power Electronics Center, 1989.

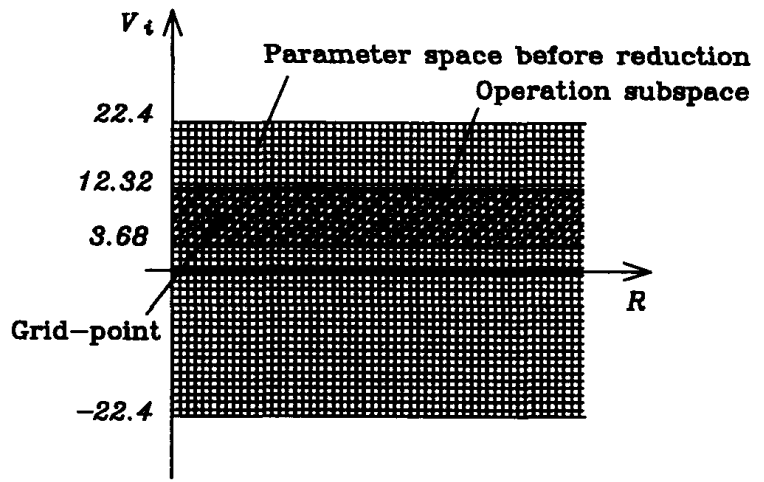

Fig. 4 Operation subspace for the grid-point of $R=5 \Omega$ and $V_{i}=8 \mathrm{~V}$.

[5] F. H. F. Leung, P. K. S. Tam and C. K. Li, "The Control of Switching Dc-dc Converters - A General LQR Problem", IEEE Trans. Ind. Electron., vol. 38, pp. 65-71, Feb. 1991.

[6] R. D. Middlebrook and S. Cuk, "A General Unified Approach to Modelling Switching-converter Power Stages", in PESC '76 Record, 1976, pp. 18-34.

[7] P. R. K. Chetty, "Modelling and Design of Switching Regulators", IEEE Trans. Aerospace Elec. Syst., vol. AES-18, no. 3, pp. 333-344, May 1982.

[8] F. H. F. Leung, P. K. S. Tam and C. K. Li, "An Improved LQR-based Controller for Switching Dc-dc Converters", IEEE Trans. Ind. Electron., vol. 40, no. 5, pp. 521-528, Oct. 1993.

[9] B. C. Kuo, Automatic Control Systems (6th ed.). Eaglewood Cliffs, NJ: Prentice-Hall, 1991.

[10] D. Kaesbauer and J. Ackermann, "The Distance from Stability or $\Gamma$ stability Boundaries", in Proceedings of the IIth IFAC World Congress: Automatic Control in the Service of Mankind, 1990, pp. 130-134.

[11] A. T. Fam and J. S. Meditch, "A Canonical Parameter Space for Linear Systems Design", IEEE Trans. Automat. Contr., vol. AC-23, no. 3, pp. 454-458, 1978

[12] D. G. Lainiotis, "Partitioning: a Unifying Framework for Adaptive Systems, I: Estimation", Proceedings of the IEEE, vol. 64, no. 8, pp. 1126-1143, 1976.

[13] C. F. Lin, Modern Navigation, Guidance, and Control Processing. Englewood Cliffs, NJ: Prentice Hall, 1991, pp. 137-138. 